\title{
3 Researc Sguare

\section{The value of circulating tumor cells with positive centromere probe 8 in the diagnosis of small pulmonary nodules.}

Caidong Liu

Nanjing First Hospital

Hongling Chen

Nanjing Medical University

Tong Sun

Nanjing Medical University https://orcid.org/0000-0002-7435-9193

Haibo Wang

Cyttelbio Corporation

\section{Baoan Chen}

Southeast University Zhongda Hospital Department of Hematology

Xuerong Wang ( $\sim$ wangxrwn@hotmail.com )

\section{Research}

Keywords: circulating tumor cells, small pulmonary nodules, EpCAM, CD45, FISH

Posted Date: March 24th, 2020

DOI: https://doi.org/10.21203/rs.3.rs-18961/v1

License: (c) (i) This work is licensed under a Creative Commons Attribution 4.0 International License.

Read Full License

Version of Record: A version of this preprint was published at Translational Oncology on May 1st, 2021. See the published version at https://doi.org/10.1016/j.tranon.2021.101052. 


\section{Abstract}

Background Circulating cancer cells (CTCs) provide opportunities for early diagnosis and evaluation of cancer stage as a more acceptable non-invasive liquid biopsy. The advanced development and use of CTCs for diagnosis or prognosis just started in recent years.

Methods Fifty three patients, diagnosed as SPNs with a diameter less than $30 \mathrm{~mm}$ by CT examination, was enrolled for statistical analysis based on their CTCs level, CT examination features, serum tumor marker concentrations, and histopathological characteristics. Centromere probe 8 (CEP8) was utilized as a marker for CTCs determination.

Results The CTCs level was significantly different between malignant and benign SPNs, as well as between early ( $0 / \mathrm{la})$ and advanced $(\mathrm{Ib} / \mathrm{II} / \mathrm{III})$ stages of lung cancer. For the malignancy judgment and the pTNM stage diagnosis of SPNs, ROC analysis results showed that combined use of CTCs level or density feature of CT morphology significant improved diagnostic effect compared to use of these two markers solely. Moreover, in bigger, single, and solid SPNs based on CT morphology, the CTCs level significantly correlated with the malignant histopathology. Additionally, triple staining (CEP8, EpCAM and CKs) results using samples from 22 out of 53 patients showed that more CTCs was detected when CEP8 was used as a marker.

Conclusion The CTCs determined by CEP8 positive would be a potential adjuvant diagnostic marker for the malignance and stage of lung cancer for patients with SPNs.

\section{Introduction}

Lung cancer is the most lethal cancer type in the world [1]. Non-small cell lung carcinoma (NSCLC) is the major type of lung cancer. It mainly contains pathologically adenocarcinomas and squamous cell carcinomas. The 5 -year survival rate of NSCLC is only approximately $19 \%$ for the reason that most of the patients were diagnosed at advanced stage [1]. However, early diagnosis and treatment can significantly increase the survival rate and improve the prognosis of the disease [2]. For instance, the 5-year disease-

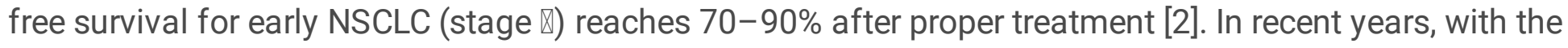
prevalence of chest computed tomography (CT) examination, millions of patients are diagnosed as small pulmonary nodules (SPNs), which are smaller than $30 \mathrm{~mm}$ (the largest diameter), solitary or multiple,ground-glass or partial-solid nodules [3, 4]. However, only approximately $30 \%$ of such SPNs are malignant lung cancer [5]. Most of them are benign diseases, such as usual interstitial pneumonia, idiopathic pulmonary fibrosis, tuberculosis [6]. There are several guidelines for management of SPNs, such as optimized surgical resection and CT surveillance, which significantly increases the survival rate; however, it also results in overtreatment, radiation exposure, or increases the risk of cancer [7]. For example, in the management of nodules, noninvasive adenocarcinoma (AIS and MIA) is considered to be indolent and subjected to sublobar resection, which preserves the function of the lung, while invasive 
adenocarcinoma $(I A)$ is different $[8,9]$. Therefore, identifying the malignant SPNs and further distinguishing the stage and invasiveness of lung cancer could dramatically benefit the patients.

Circulating cancer cells (CTCs) are cancer cells circulating in the blood that detached from original or metastatic tumors and invaded to the vascular system [10]. They are the main causes of cancer metastasis and cancer-related death [11]. However, they also provide opportunities for early diagnosis and evaluation of cancer stage as a more acceptable non-invasive liquid biopsy. The study of CTCs began more than a hundred year ago, but the advanced development and use of CTCs for diagnosis or prognosis just started in recent years $[12,13]$. Even though CellSearch is the only method that approved by FDA (Food and Drug Administration) of U.S. for clinical determination of CTCs, there are many methods and technologies used in academic study and clinical adjuvant diagnosis [14]. Commonly, CD45 positive cells are depleted from peripheral blood cells as lymphocytes. Epithelial markers, such as EpCAM (epithelial cancer associated marker) and CKs (cytokeratins), positive cells are determined as CTCs from epithelial-originated cancers, including lung and breast cancer $[15,16]$. Folate receptor, another marker for CTCs of lung cancer, has also been well studied [17]. Very recently, Zhou et al. reported the correlation between folate receptor-positive CTCs with indeterminate lung nodules and suggested its prognostic values [4]. Currently, no method is fully satisfied for diagnosis and prognosis. Thus, specific markers are urgently needed for determination CTCs from different cancer types. To date, numerous studies have shown the correlation between CTCs and cancer stages, invasiveness, and metastasis, including lung cancer $[18,19]$. However, for the reason that CTCs are extremely rare in NSCLC, whether CTCs could be detected in the peripheral blood of SPNs patients are quite unknown [20]. Therefore, collecting CTCs and analyzing characteristics of these CTCs would greatly improve the sensitivity and specificity of the lung cancer diagnosis, and provide proper subsequent therapeutic strategies.

In this study, we collected patients diagnosed as SPNs by CT examination and expected to undergo surgery. We detected the CTCs in their peripheral venous blood before lung resection using centromere probe 8 (CEP8) as the selection marker. After we collected the pathological diagnosis of these SPNs, such as benignancy, malignancy, cancer type, stage and invasiveness, the level of CTCs were subjected to statistical analysis for their correlation with CT, serum markers, and histopathological characteristics. We also compared the positive rate of different markers for defining CTCs, including CEP8, EpCAM, and pan CKs. Our findings suggest that CTCs could be enriched from $3.2 \mathrm{~mL}$ peripheral blood of SPNs patients, and its level is correlated with the histopathological characteristics of SPNs. CTCs is a potential adjuvant diagnostic marker for SPNs.

\section{Materials And Methods}

\section{Patients}

This study included 53 patients with SPNs detected by computed tomography (CT) examination at Nanjing First Hospital from May to November in 2019. CT-detected single or multiple lung nodules with the diameter $\leq 30 \mathrm{~mm}$ were defined as SPNs. Diagnosis was determined by histopathological examine of 
tissues from lung surgery. Forty-one out of 53 patients were malignant SPNs, and most of which (38/41) were adenocarcinomas. Twelve out 53 patients were benign SPNs, which include fibrosis, hyperplasia and tuberculosis. Patients with or without pulmonary clinical symptoms, such as cough, chest pain were included. Patients with chemotherapy, radiotherapy, and lung cancer-related disease or surgery were excluded. The histological types of NSCLC and the diagnosis of adenocarcinoma of in situ (AIS), minimally invasive adenocarcinoma (MIA), and invasive adenocarcinoma (IA) of the lung were determined according to $\mathrm{WHO}$ classification of tumors of the lung. The pathological tumor nodal metastasis (pTNM) stage was determined according to AJCC stage manual ( $8^{\text {th }}$. Version). Histopathology diagnosis was obtained and determined by two pathologists. The study was approved by the Ethics Committee of Nanjing Medical University. Informed consent was obtained from all the participants.

Isolation and identification of CTCs

Peripheral venous blood $(3.2 \mathrm{~mL})$ from each patient before lung surgery was collected in customized Acid Citrate Dextrose (ACD)-anticoagulant tubes (Becton Dickinson, NJ, USA). CTCs was enriched and determined by the Cyttel method following the instruction of the manufacturers (Cyttel, Jiangsu, China) as previously reported [19]. Briefly, red blood cell (RBC) lysis buffer was used to deplete the RBCs from the whole blood. The leukocytes were removed by centrifugation after incubated with anti-CD45 antibodyconjugated immuno-magnetic beads. The resulting solution containing CTCs was smeared on the slide for subsequent analysis.

Centromere probe 8 (CEP8) in the cells on the slide was detected by fluorescence in situ hybridization (FISH) method as previously reported [21]. CEP8 staining indicated the copy numbers of chromosome 8 in the cell. Then, Alexa Fluor 594 conjugated anti-human CD45 antibody (Cyttel, Jiangsu, China) and DAPI (Vector Laboratories, Burlingame, CA, USA) were used to stain leukocytes and cell nucleus. Cells with features of CEP8 $\geq 2 / \mathrm{CD} 45 / \mathrm{DAPI}{ }^{+}$were considered CTCs. Identification of CTCs was performed by independent researchers in a blinded manner [18].

Some slides (22/53) were subjected to immunofluorescence for two more tumor markers, EpCAM and pan CKs for further analysis. Cells on the slides were incubated with primary anti-EpCAM (BAF960, R\&D system) or anti-pan Cytoceratins (pan CKs) (ab7753, abcam) antibodies, followed by secondary antibody Alexa Fluor 488 or Alexa Fluor 555 (ab150129 and ab105106, abcam). Fluorescent images were captured with an Olympus BX63 microscope using the IMSTAR high content screening device (IMSTARSA, France).

Identification of SPNs by CT

The features of SPNs were classified into different groups, small $(<15 \mathrm{~mm})$ and big $(\geq 15 \mathrm{~mm}, \leq 30 \mathrm{~mm})$ based on the nodule size, single $(n=1)$ and multiple $(n>1)$ based on the nodule number, or solid (high density, cord-like appearance, or with globular mass) and non-solid (low density, or ground glass-like appearance) based on the nodule density. Two independent radiologists read the CT image. 
Serum samples (from peripheral venous blood) were collected in anticoagulant-free blood-collecting tubes. Tumor markers, such as CEA, CYFRA 21-1, and AFP, were detected via the CMIA (chemiluminescence microparticle immunoassays) methods (Reagent kit \#7K68, 2P55, 3P36) using ARCHITECT-i2000sr (Abbott Laboratories, USA). CA 19-9 was detected via automatic electrochemiluminescence immunoassay (Reagent kit \#11776193) using Cobas e602 (Roche Ltd, Germen).

Statistical analysis

The Mann-Whitney Utest and the Student's $t$ test were used to compare different groups. The chi-square tests or the Fisher's exact tests were used to compare the categorical variables. The ROC (receiver operating characteristic) analysis was performed and the AUC (area under the curve) were calculated to assess the histopathology of SPNs. $p<0.05$ was considered as significant. The data were analyzed using SPSS 18.0 and Prism 6.0 software.

\section{Results}

The level of CTCs was correlated with malignant SPNs.

There are amounts of studies detected CTCs in advanced cancer patients, including lung cancer. However, whether CTCs could be detected from the peripheral venous blood of SPNs patients is unknown. We first enriched the CTCs from patients diagnosed as SPNs by CT examination. We collected the histopathologic diagnosis of the SPNs if the patient underwent surgery. We also examined the serum tumor markers before the surgery. Fifty-three patients which successfully completed CTCs, CT, serum markers, and histopathology examinations were included for the subsequent statistically analysis. The demographic and clinic pathologic characteristics of the patients were displayed in Supplementary table S1.

We found that $41 / 53$ patients were lung cancer, and most of which (38/41) were adenocarcinomas. Since the invasiveness of adenocarcinomas provide critical guidelines for the resection strategy and is correlated with the prognosis, we classified the patients into 3 groups, AIS, MIA, and IA. We also divided

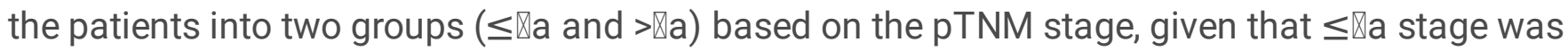
recommended for limited surgical resection, such as segmentectomy, sublobar or lobectomy resection, which would both increase the survival rate and maximally preserve the lung function [22-25]. Twelve out of 53 patients were benign pulmonary diseases, such as fibrosis, hyperplasia, and tuberculosis. The representative hematoxylin \& eosin (H\&E) staining of these tissues were shown in Fig. 1. 
Then, we analyzed the correlation between CTCs level and malignant SPNs. CEP8+/ CD45 / DAPI + were determined as CTCs. Representative staining was shown in Fig. 2A. The level of CTCs was presented by the number of CTCs in each sample. We found that the CTCs level was significantly higher in malignant SPNs than benign SPNs by Mann-Whitey U test $(p<0.05)$ (Fig. 2B). Similarly, the average CTCs numbers of malignant SPNs were significantly higher than benign SPNs by the student $t$ test (Supplementary

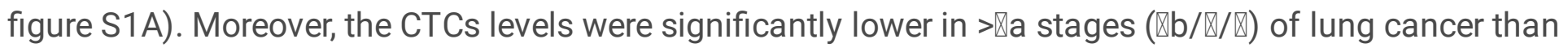

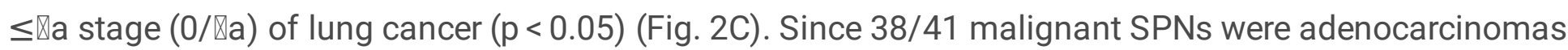
(Supplementary figure S1B), we analyzed the correlation between the CTCs levels and cancer invasiveness. However, no significant difference of the CTC levels was observed among AIS, MIA and IA groups (Fig. 2D). Considering the limited sample size, we pooled MIA and AIS to make up noninvasive group compare with invasive group (IA), since MIA was considered as noninvasiveness pathologically. We did not observe any statistical difference in the CTCs levels (Supplementary figure S1C).

Furthermore, the ROC analysis showed that the sensitivity and specificity for the CTCs as a diagnostic marker to distinguish malignant SPNs from benign SPNs were $92.7 \%$ and $50 \%$, respectively (Fig. 2E, Table 1). The area under curve (AUC) of the CTC levels was 0.713 with a statistical significance, indicating it would be an independent diagnostic marker for malignancy $(p<0.05)$. However, the CTCs levels showed no significant difference to distinguish $\leq$ \a stage lung cancer from $>$ \a stage lung cancer (Fig. 2F), as well as to distinguish invasive from non-invasive SPNs (Supplementary figure S1D) and adenocarcinoma from other types (Supplementary figure S1B and S1E). These findings suggest that the levels of CTCs were increased in malignant SPNs compared to benign SPNs, albeit very early stage of lung cancer released more CTCs than late stage disease. The level of CTCs would serve as a potential liquid biopsy marker for the diagnosis of malignant SPNs.

Table 1

results of ROC analysis (malignant versus benign)

\begin{tabular}{|llllll|}
\hline & AUC $(95 \% \mathrm{Cl})$ & p value & Cut-off value & Sensitivity (\%) & Specificity (\%) \\
\hline CTCs & $0.713(0.525,0.902)$ & 0.026 & 0.5 & 92.7 & 50.0 \\
\hline Size & $0.462(0.274,0.651)$ & 0.694 & 0.5 & 34.1 & 58.3 \\
\hline Density & $0.702(0.551,0.853)$ & 0.034 & 0.5 & 48.8 & 91.7 \\
\hline CTCs + Density & $0.726(0.537,0.914)$ & 0.018 & 0.5 & 95.1 & 50.0 \\
\hline
\end{tabular}

Combined use of CTCs level and density features of the CT examination identified malignant SPNs.

Currently, with the growing use of CT examination, especially thin-section CT scan, millions of SPNs were detected annually; however, only $30 \%$ were finally identified as malignant. Thus, more adjuvant diagnostic methods were urgently needed. We firstly analyzed the correlation between the CT morphology and the clinicopathological characteristics of SPNs, based on the most frequently used three criteria, such assize (the largest diameter), number, and density of the nodules. As shown in Table 1, no significant correlation 
was observed between size and malignancy of the nodules, neither between the nodule number and malignacy. However, the density of the nodules showed significant positive correlation with the malignancy (Table 2). Statistical analysis also showed that there had no correlation between size, number, or density of the nodules and the cancer stages, neither any correlation with cancer types were observed (Supplementary table S2). Moreover, the number of the nodules displayed significant correlation with invasiveness of adenocarcinomas, but no correlation exhibited between the size and the invasiveness (Supplementary table S3). Furthermore, no obvious correlation was observed between the density and the invasiveness (Supplementary table S3). Considering this may be caused by limited sample size, we pooled AIS and MIA together and observed a significant positive correlation between density and invasiveness (Supplementary table S4). These findings were consistent with other reports that the high density of the nodules was an important indicator for malignancy.

Table 2

the correlation between CT morphology and histology of SPNs

\begin{tabular}{|c|c|c|c|c|c|}
\hline \multirow[t]{2}{*}{ CT parameters } & \multirow[t]{2}{*}{$n$} & \multicolumn{2}{|l|}{ Histology } & \multirow[t]{2}{*}{$\mathrm{X}^{2}$ value } & \multirow[t]{2}{*}{$\mathrm{p}$ value } \\
\hline & & Malignant & Benign & & \\
\hline \multicolumn{6}{|l|}{ Size } \\
\hline$<15 \mathrm{~mm}$ & 34 & 27 & 7 & 0.018 & 0.892 \\
\hline$\geq 15 \mathrm{~mm}$ & 19 & 14 & 5 & & \\
\hline \multicolumn{6}{|l|}{ Number } \\
\hline Single & 30 & 23 & 7 & 0.019 & 0.891 \\
\hline Multiple & 23 & 18 & 5 & & \\
\hline \multicolumn{6}{|l|}{ Density } \\
\hline Solid & 32 & 21 & 11 & 4.77 & $0.029^{a}$ \\
\hline Non-solid & 21 & 20 & 1 & & \\
\hline
\end{tabular}

Then, we analyzed the correlation between the CTCs levels and CT examination. We did not observe any significant difference of the CTCs level between the small $(<15 \mathrm{~mm})$ and big $(\geq 15 \mathrm{~mm})$ SPNs group based on the CT examination (Fig. 3A). We investigated the prediction of SPNs malignancy by considering both the CTCs level and the size of CT morphology. As shown in Fig. 3B, the CTCs level was significantly higher in malignant than in benign SPNs in big SPNs group, but not in small group.

Moreover, although no significant difference in CTCs level was exhibited between the number of single and multiple groups, the level of CTCs showed a significant increase in malignancy compared with benign SPNs when only single SPNs are considered (Fig. 3C and D). No difference of the CTCs level was 
observed between solid and non-solid group, albeit the level of CTCs was obviously higher in malignancy than benign SPNs when only solid SPNs are considered (Fig. 3E and F). We did not analyze non-solid group for the reason of lacking enough sample number in benign SPNs group.

Next, we analyzed the sensitivity and specificity of the CTCs level combined with CT characteristics for prediction of malignancy of SPNs by ROC analysis. The AUC of the density, the CTCs levels, but not the size, showed statistical significance (Fig. 3G). Moreover, combined use of the CTCs levels and the density features exhibited higher AUC than each single item. As shown in Table 1, the CTCs showed the highest sensitivity (92.7\%) and the density showed the highest specificity (91.7\%), when they were solely used for identifying malignancy. Combined use of these two features did further increase the sensitivity but not the specificity.

Additionally, we analyzed the CTCs level, size and density features of the nodules in differentiating the $\leq$ Wa stages from $>$ Wa stages of lung cancer. The ROC curve showed that the AUC of each feature did not display any statistical significance; however, combined use of CTCs level and the density increased the AUC to 0.735 compared to each single one ( 0.61 for CTCs and 0.648 for density) (Fig. $3 \mathrm{H}$ ). As shown in Table 3, combined use of CTCs level and density significantly correlates with SPNs stage $(p=0.041)$ and, with a sensitivity of $97 \%$ and specificity of $50 \%$, this combined diagnostic strategy improved the overall diagnostic effect compared with when solely CTCs level or density was considered.

Table 3

results of ROC analysis ( $\leq$ 『a stages versus $>$ \a stages)

\begin{tabular}{|llllll|}
\hline & AUC $(\mathbf{9 5 \%}$ Cl) & p value & Cut-off value & Sensitivity (\%) & Specificity (\%) \\
\hline CTCs & $0.610(0.369,0.851)$ & 0.34 & 0.5 & 97.0 & 25.0 \\
\hline Size & $0.324(0.128,0.540)$ & 0.126 & 0.5 & 27.3 & 37.5 \\
\hline Density & $0.648(0.441,0.855)$ & 0.199 & 0.5 & 54.5 & 75.0 \\
\hline CTCs + Density & $0.735(0.369,0.851)$ & 0.041 & 0.5 & 97.0 & 50.0 \\
\hline
\end{tabular}

These findings suggest that combined use of the density feature of the CT examination and the CTCs level could be developed as a potent adjuvant quality and stage diagnosis of SPNs.

The serum tumor markers did not show any correlation with malignant SPNs, but the CEA levels were correlated with the CTCs levels.

We also examined the serum tumor makers of the SPNs patients. To our surprise, most of the patients showed negative results based on the normal criteria $(<5.0 \mathrm{ng} / \mathrm{mL}$ for CEA, $<7.0 \mathrm{ng} / \mathrm{mL}$ for CYFRA $21-1$, $<7.29 \mathrm{ng} / \mathrm{mL}$ for AFP, and $<27.0 \mathrm{Unit} / \mathrm{mL}$ for CA $19-9$ ), possible due to most of the subjects in this study are early lung cancer or benign diseases. Moreover, the average concentrations of the each marker did not show any significant difference between malignant and benign SPNs groups (Fig. 4A). We analyzed these markers between the two groups in CTCs positive or negative patients. As shown in 
Fig. 4B, among the 4 serum markers, only the CEA concentrations exhibited difference between CTC + and CTC- groups. These findings indicate that the concentration of CEA would be different if the patient has cTCs.

The comparison the CEP8, EpCAM, and pan CKs for CTCs identification.

Currently, the specific markers of CTCs have not been well identified. Even the same population could show opposite results for CTCs levels when different methods were used [26, 27]. In this study, CEP8 positive cells by FISH assay was defined as the CTCs. We then compared other frequently used markers such as EpCAM and pan CKs for the detection of CTCs using immunofluescence staining in the same slides following CEP8 examine (Fig. 2A). We found that among the 22 samples that successfully completed all three markers staining, only 5 patients (1 slide for each patients) had dual positive cells $\mathrm{EpCAM+/pan} \mathrm{CKs} \mathrm{+} \mathrm{with} \mathrm{a} \mathrm{detection} \mathrm{rate} \mathrm{of} 22.7 \%$, which was consistent with others report [28]. And there were some cells showed triple positive staining. The level of CTCs showed no significant difference between pan CKs and EpCAM positive cells. However, CEP8 positive cell numbers were higher than EpCAM or pan CKs with a statistical significance (Supplementary figure S2). These findings indicated that different markers detected different subpopulations of CTCs due to the heterogeneity of cancer cells.

\section{Discussion}

Lung cancer is the most prevalent cancer and the leading cause of cancer-related death worldwide [29]. Early diagnosis and treatment dramatically increased the survival rate of the patient [4]. So, early diagnosis of lung cancer attracted great research interests and developed fast in recent years. CTCs could be enriched from peripheral blood, a kind of fluid biopsy, noninvasive and more acceptable for patients [30]. But it is difficult to develop CTCs as a diagnostic marker, because the number of CTCs was extremely low in the peripheral blood. Different detection methods obtained different CTC numbers in different volumes of blood [31]. Recently, Teixeira et al. enriched and sequencing the CTCs collected from $7.5 \mathrm{~mL}$ peripheral blood of patients with pre-invasive squamous cell lung cancer lesions using Cellsearch system [32]. Even though large amount of studies reported the correlation of CTCs with different types of cancers or different clinicopathological characteristics of cancer, whether CTCs could be enriched from SPNs, a very early stage of cancer, are quite unknown. Recently FR+ (folate receptor) CTCs were detected and used to distinguish invasive and preinvasive lung adenocarcinomas [4]. In this study, we for the first time detected CTCs from $3.2 \mathrm{~mL}$ of the peripheral venous blood of SPNs patients. It showed that the level of CTCs was higher in malignant group than benign group. Furthermore, the ROC analysis also showed that the the method of measuring CTCs level was able to distinguish malignant SPNs from benign SPNs with high sensitivity. These findings suggest that CTCs level could be developed as a potential adjuvant diagnostic marker for SPNs. To our surprise, more CTCs were detected in very early stage (0/囚a) of lung cancer than advanced stages $(\nabla b / \nabla / \nabla)$. Since CTCs are escaped cancer cells that invaded to the circulating system and survived, we suspected that in the advanced stages of lung cancer, the tumor microenvironment was formed, therefore, CTCs was stuck in the tumor. It is possible a sample bias because most of the SPNs patients were in early stages. 
Currently, most of the SPNs were first identified by CT examination. With the prevalence of chest CT, especially thin-section $\mathrm{CT}$, the detection rate of small pulmonary nodules $(\leq 30 \mathrm{~mm})$ significant increased. However, most of the patients were lately diagnosed as non-cancer-related diseases, just inflammation, hyperplasia, fibrosis, tuberculosis, or even a stress reaction [33]. These patients take the CT examination for some light clinical symptoms, such as cough, chest pain, minor uncomfortable, or merely for annual physical examination. Thus, as soon as be diagnosed as SPNs, which indicated the risk of lung cancer, they were subjected to several choices, such as CT surveillance, CT-guided biopsy, or direct surgery. Recently, diagnosis of cancer by CT morphology was greatly developed. Various radiomics nomogram made great progress in identifying malignancy from benign SPNs, determining the invasiveness of lung adenocarcinomas, and even distinguishing ground-glass nodules less than $10 \mathrm{~mm}$ $[34,35]$. These progresses provided critical guidelines for appropriate treatment which resulted in obviously improvement for the lung cancer prognosis [36]. In the current study, we also observed that the density of the nodules, but not the size of the nodules, distinguished malignant SPNs from benign SPNs according to AUC in the ROS curve. Combined use of CTCs and density increased AUC significantly.

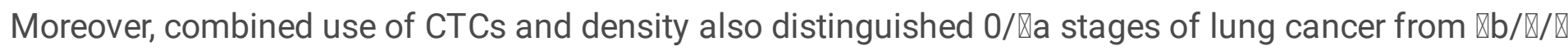
stages. On another hand, we observed significant increase in the CTCs level of patients with the nodule size bigger than $15 \mathrm{~mm}$ and the patients with solitary nodules. These findings indicate that when a patient was diagnosed as SPNs by CT examination, elevated CTCs level suggested malignancy if the nodules was bigger than $15 \mathrm{~mm}$ or the nodules was solid. Therefore, CTCs is a potential adjuvant diagnostic marker in collaboration with CT examination.

Another issue of this study is that the patients who were finally included in the study were those who took the surgery and diagnosed by histopathology, based on the CT morphological changes. Surgeries were suggested only for those with risky CT morphological changes. In the CT diagnositic process of identifying malignancy, the density feature gained higher weight than other features, which would cause some bias in this study. This might also be the reason that combined use of CTCs and density did not further increase the specificity of malignancy diagnosis in the ROC analysis, since that the density exhibited the highest specificity alone. Lindsay et al reported that the CTCs could be an independent prognostic marker for advanced NSCLCs based on a multicenter, large size investigation [37]. We are carefully to say that it could be an independent diagnostic marker for SPNs based on our findings that the CTCs level was obviously correlation with malignancy, because of the limited samples size and the possible bias in the study.

Specific serum tumor markers were helpful in increasing the sensitivity of malignant diagnosis; however, the specificity is not satisfied. Some serum markers are indicative for the origin of the cancer types, such as CEA for colon cancer, AFP for live cancer, and CYFRA 21 - 1 for lung cancer [38]. In this study, we did not observed any significant alterations of serum markers based on the criteria used for clinical diagnosis. Neither did us observed any difference of average serum concentrations between malignant and benign groups. We identified increased CEA concentration in CTCs positive group of SPNs, indicating that these two factors may be correlated. These findings indicate that changes of the serum tumor markers might not be significant in very early stage of lung cancer. 
We also compared several CTCs markers in this study. Even though EpCAM positive was acknowledged by most of the researchers for CTCs, and CellSearch was the only approved method for detection of CTCs, finding better methods for CTCs detection are still on the way [39]. Firstly, EpCAM was a highly expressed marker in epithelial cells, this would cause the issue that EpCAM positive cells may include some circulating epithelial cells and endothelial cells dropped physiologically into the blood stream [40]. Secondly, some cancer cells did not express EpCAM due to heterogeneity or epithelial-mesenchymal transition (EMT), a process that cancer cells lose their epithelial features to increase migratory or invasive capacities [41]. Therefore, not all cancer cells expression EpCAM. Same issues existed for using CKs as a marker for CTCs [16]. Folate receptor (FR) positive cells as a CTCs marker was reported to distinguish malignant from benign diseases $[4,42]$. Compared with FR (folate receptor) and MTD (maximum tumour diameter) combined system reported [4], our CEP8 and CT combined SPNs diagnostic system exhibited much higher sensitivity (95.1\% versus 78.6\%-82.7\%). In addition, FR and MTD combined system could only differentiate noninvasive cancers from invasive cancers while our system could distinguish the nature of SPNs, i.e. between benignancy and malignancy, which would be used as a further upstream diagnostic guidance in the treatment of SPNs. In this study, we used CEP8 as a CTCs marker. Notably, we successfully stained 22 samples with CEP8, EpCAM, and pan CKs. Even though this study was the first to use Cyttee method for CTCs detection in SPNs, we observed that the level of CEP8 + CTCs was higher than EpCAM + and CKs + cells. However, there also observed some triple positive cells. The detection rate of cells with dual EpCAM+/CKs + staining (22.7\%, 5 out of 22 patients) was consistent with other reports (approximately 20\%) [28]. These findings suggest that different subpopulations of CTCs express different markers, and more new markers for identifying CTCs are urgently needed.

In summary, we detected the CTCs using CEP8 as a marker in the $3.2 \mathrm{~mL}$ peripheral venous blood from patients with SPNs diagnosed by CT examination. The elevated level of CTCs was correlated with the malignancy and stages of SPNs. Combined use of CTCs and density feature of CT morphogical diagnosis increased the sensitivity in distinguishing malignant SPNs. Our findings suggest that CTCs level combined with CT diagnosis would be used as a novel marker for determining the nature of SPNs at an early stage.

\section{Declarations}

Ethics approval and consent to participate

The study was approved by the Ethics Committee of Nanjing Medical University.

Consent for publication

Informed consent was obtained from all the participants.

Availability of data and materials 
The datasets used and/or analysed during the current study are available from the corresponding author on reasonable request.

Competing interests

The authors declare no competing of interest.

Funding

This work is supported by the National Natural Science Foundation of China under Grant No. 81473241, 81972763 for X, Wang, 81702882 for T, Sun; Key Laboratory of Human Functional Genomics of Jiangsu Province (X, Wang and T, Sun); Science and Technology Department of Jiangsu Province under Grant No. BK20171056 for T, Sun; Jiangsu Social Development Project under Grant No. BE2018711 for B, Chen.

Authors' Contributions

$\mathrm{CL}, \mathrm{HC}$ and TS conducted sample collection, the experiments, and the statistical analysis. $\mathrm{BC}$ and $\mathrm{HW}$ were involved in study design and manuscript writing. XW were responsible for study design, data analysis, and manuscript writing. All authors reviewed the manuscript.

Authors' information

${ }^{1}$ Department of Laboratory Medicine, Nanjing First Hospital, Nanjing Medical University, Nanjing, Jiangsu Province, China. ${ }^{2}$ Department of Pharmacology, Nanjing Medical University, Nanjing, Jiangsu Province, China. ${ }^{3}$ Key Laboratory of Human Functional Genomics of Jiangsu Province, Nanjing Medical University, Nanjing, Jiangsu Province, China. ${ }^{4}$ Cyttelbio Corporation, Beijing, China. ${ }^{5}$ Department of Hematology and Oncology, Zhongda Hospital, School of Medicine, Southeast University, Nanjing, Jiangsu Province, China.

\section{References}

1. Siegel RL, Miller KD, Jemal A: Cancer statistics, 2020. CA Cancer J Clin 2020, 70:7-30.

2. Blandin Knight S, Crosbie PA, Balata H, Chudziak J, Hussell T, Dive C: Progress and prospects of early detection in lung cancer. Open Biol 2017, 7.

3. Gould MK, Donington J, Lynch WR, Mazzone PJ, Midthun DE, Naidich DP, Wiener RS: Evaluation of individuals with pulmonary nodules: when is it lung cancer? Diagnosis and management of lung cancer, 3rd ed: American College of Chest Physicians evidence-based clinical practice guidelines. Chest 2013, 143:e93S-e120S.

4. Zhou Q, Geng Q, Wang L, Huang J, Liao M, Li Y, Ding Z, Yang S, Zhao H, Shen Q, et al: Value of folate receptor-positive circulating tumour cells in the clinical management of indeterminate lung nodules: A non-invasive biomarker for predicting malignancy and tumour invasiveness. EBioMedicine 2019, 41:236-243. 
5. She Y, Zhao L, Dai C, Ren Y, Jiang G, Xie H, Zhu H, Sun X, Yang P, Chen Y, et al: Development and validation of a nomogram to estimate the pretest probability of cancer in Chinese patients with solid solitary pulmonary nodules: A multi-institutional study. J Surg Onco/2017, 116:756-762.

6. Furman AM, Dit Yafawi JZ, Soubani AO: An update on the evaluation and management of small pulmonary nodules. Future Oncol 2013, 9:855-865.

7. Jin X, Chen Y, Chen H, Fei S, Chen D, Cai X, Liu L, Lin B, Su H, Zhao L, et al: Evaluation of TumorDerived Exosomal miRNA as Potential Diagnostic Biomarkers for Early-Stage Non-Small Cell Lung Cancer Using Next-Generation Sequencing. Clin Cancer Res 2017, 23:5311-5319.

8. She Y, Zhang L, Zhu H, Dai C, Xie D, Xie H, Zhang W, Zhao L, Zou L, Fei K, et al: The predictive value of CT-based radiomics in differentiating indolent from invasive lung adenocarcinoma in patients with pulmonary nodules. Eur Radio/ 2018, 28:5121-5128.

9. Kadota K, Villena-Vargas J, Yoshizawa A, Motoi N, Sima CS, Riely GJ, Rusch VW, Adusumilli PS, Travis WD: Prognostic significance of adenocarcinoma in situ, minimally invasive adenocarcinoma, and nonmucinous lepidic predominant invasive adenocarcinoma of the lung in patients with stage I disease. Am J Surg Pathol 2014, 38:448-460.

10. Zou D, Cui D: Advances in isolation and detection of circulating tumor cells based on microfluidics. Cancer Biol Med 2018, 15:335-353.

11. Massague J, Obenauf AC: Metastatic colonization by circulating tumour cells. Nature 2016, 529:298306.

12. Paget S: The distribution of secondary growths in cancer of the breast. 1889. Cancer Metastasis Rev 1989, 8:98-101.

13. Maheswaran S, Haber DA: Circulating tumor cells: a window into cancer biology and metastasis. Curr Opin Genet Dev 2010, 20:96-99.

14. Shen Z, Wu A, Chen X: Current detection technologies for circulating tumor cells. Chem Soc Rev 2017, 46:2038-2056.

15. Weigelt B, Ng CK, Shen R, Popova T, Schizas M, Natrajan R, Mariani O, Stern MH, Norton L, VincentSalomon A, Reis-Filho JS: Metaplastic breast carcinomas display genomic and transcriptomic heterogeneity [corrected]. Mod Patho/ 2015, 28:340-351.

16. Alix-Panabieres C, Pantel K: Challenges in circulating tumour cell research. Nat Rev Cancer 2014, 14:623-631.

17. Nunez MI, Behrens C, Woods DM, Lin H, Suraokar M, Kadara H, Hofstetter W, Kalhor N, Lee JJ, Franklin W, et al: High expression of folate receptor alpha in lung cancer correlates with adenocarcinoma histology and EGFR [corrected] mutation. J Thorac Onco/ 2012, 7:833-840.

18. Ding C, Zhou X, Xu C, Chen J, Ju S, Chen T, Liang Z, Cui Z, Li C, Zhao J: Circulating tumor cell levels and carcinoembryonic antigen: An improved diagnostic method for lung adenocarcinoma. Thorac Cancer 2018, 9:1413-1420.

19. Li Y, Tian X, Gao L, Jiang X, Fu R, Zhang T, Ren T, Hu P, Wu Y, Zhao P, Yang D: Clinical significance of circulating tumor cells and tumor markers in the diagnosis of lung cancer. Cancer Med 2019, 8:3782- 
3792.

20. Hofman V, Heeke S, Marquette CH, llie M, Hofman P: Circulating Tumor Cell Detection in Lung Cancer: But to What End? Cancers (Basel) 2019, 11.

21. Li Y, Ma G, Zhao P, Fu R, Gao L, Jiang X, Hu P, Ren T, Wu Y, Wang Z, et al: Improvement of sensitive and specific detection of circulating tumor cells using negative enrichment and immunostainingFISH. Clin Chim Acta 2018, 485:95-102.

22. Ginsberg RJ, Rubinstein LV: Randomized trial of lobectomy versus limited resection for T1 N0 nonsmall cell lung cancer. Lung Cancer Study Group. Ann Thorac Surg 1995, 60:615-622; discussion 622-613.

23. Yoshizawa A, Motoi N, Riely GJ, Sima CS, Gerald WL, Kris MG, Park BJ, Rusch VW, Travis WD: Impact of proposed IASLC/ATS/ERS classification of lung adenocarcinoma: prognostic subgroups and implications for further revision of staging based on analysis of 514 stage I cases. Mod Pathol 2011, 24:653-664.

24. Altorki NK, Yip R, Hanaoka T, Bauer T, Aye R, Kohman L, Sheppard B, Thurer R, Andaz S, Smith M, et al: Sublobar resection is equivalent to lobectomy for clinical stage $1 A$ lung cancer in solid nodules. $J$ Thorac Cardiovasc Surg 2014, 147:754-762; Discussion 762-754.

25. Harada H, Okada M, Sakamoto T, Matsuoka $H$, Tsubota N: Functional advantage after radical segmentectomy versus lobectomy for lung cancer. Ann Thorac Surg 2005, 80:2041-2045.

26. Farace F, Massard C, Vimond N, Drusch F, Jacques N, Billiot F, Laplanche A, Chauchereau A, Lacroix $L$, Planchard D, et al: A direct comparison of CellSearch and ISET for circulating tumour-cell detection in patients with metastatic carcinomas. Br J Cancer 2011, 105:847-853.

27. Hofman V, Ilie MI, Long E, Selva E, Bonnetaud C, Molina T, Venissac N, Mouroux J, Vielh P, Hofman P: Detection of circulating tumor cells as a prognostic factor in patients undergoing radical surgery for non-small-cell lung carcinoma: comparison of the efficacy of the CellSearch Assay and the isolation by size of epithelial tumor cell method. Int J Cancer 2011, 129:1651-1660.

28. Alix-Panabieres C, Pantel K: Clinical Applications of Circulating Tumor Cells and Circulating Tumor DNA as Liquid Biopsy. Cancer Discov 2016, 6:479-491.

29. Siegel RL, Miller KD, Jemal A: Cancer statistics, 2019. CA Cancer J Clin 2019, 69:7-34.

30. Watts G: Liquid biopsy: still early days for early detection. Lancet 2018, 391:2593-2594.

31. Zhong X, Zhang H, Zhu Y, Liang Y, Yuan Z, Li J, Li J, Li X, Jia Y, He T, et al: Circulating tumor cells in cancer patients: developments and clinical applications for immunotherapy. Mol Cancer 2020, $19: 15$.

32. Teixeira VH, Pipinikas CP, Pennycuick A, Lee-Six H, Chandrasekharan D, Beane J, Morris TJ, Karpathakis A, Feber A, Breeze CE, et al: Deciphering the genomic, epigenomic, and transcriptomic landscapes of pre-invasive lung cancer lesions. Nat Med 2019, 25:517-525.

33. Henschke Cl, Yankelevitz DF, Mirtcheva R, McGuinness G, McCauley D, Miettinen OS, Group E: CT screening for lung cancer: frequency and significance of part-solid and nonsolid nodules. AJR Am J Roentgenol 2002, 178:1053-1057. 
34. Fan L, Fang M, Li Z, Tu W, Wang S, Chen W, Tian J, Dong D, Liu S: Radiomics signature: a biomarker for the preoperative discrimination of lung invasive adenocarcinoma manifesting as a ground-glass nodule. Eur Radiol 2019, 29:889-897.

35. Zhao W, Xu Y, Yang Z, Sun Y, Li C, Jin L, Gao P, He W, Wang P, Shi H, et al: Development and validation of a radiomics nomogram for identifying invasiveness of pulmonary adenocarcinomas appearing as subcentimeter ground-glass opacity nodules. Eur J Radiol 2019, 112:161-168.

36. Mateo J, Gerlinger M, Rodrigues DN, de Bono JS: The promise of circulating tumor cell analysis in cancer management. Genome Biol 2014, 15:448.

37. Lindsay CR, Blackhall FH, Carmel A, Fernandez-Gutierrez F, Gazzaniga P, Groen HJM, Hiltermann TJN, Krebs MG, Loges S, Lopez-Lopez R, et al: EPAC-lung: pooled analysis of circulating tumour cells in advanced non-small cell lung cancer. Eur J Cancer 2019, 117:60-68.

38. Molina R, Agusti C, Mane JM, Filella X, Jo J, Joseph J, Gimenez N, Estape J, Ballesta AM: CYFRA 211 in lung cancer: comparison with CEA, CA 125, SCC and NSE serum levels. Int J Biol Markers 1994, 9:96-101.

39. Cristofanilli M, Budd GT, Ellis MJ, Stopeck A, Matera J, Miller MC, Reuben JM, Doyle GV, Allard WJ, Terstappen LW, Hayes DF: Circulating tumor cells, disease progression, and survival in metastatic breast cancer. N Engl J Med 2004, 351:781-791.

40. Krebs MG, Metcalf RL, Carter L, Brady G, Blackhall FH, Dive C: Molecular analysis of circulating tumour cells-biology and biomarkers. Nat Rev Clin Oncol 2014, 11:129-144.

41. Krebs MG, Hou JM, Sloane R, Lancashire L, Priest L, Nonaka D, Ward TH, Backen A, Clack G, Hughes $A$, et al: Analysis of circulating tumor cells in patients with non-small cell lung cancer using epithelial marker-dependent and -independent approaches. J Thorac Oncol 2012, 7:306-315.

42. Wang L, Wu C, Qiao L, Yu W, Guo Q, Zhao M, Yang G, Zhao H, Lou J: Clinical Significance of Folate Receptor-positive Circulating Tumor Cells Detected by Ligand-targeted Polymerase Chain Reaction in Lung Cancer. J Cancer 2017, 8:104-110.

\section{Figures}

\section{Figure 1}

The representative histopathology of SPNs tissues with HE staining. Magnitude, 200x. 
A

CEP8

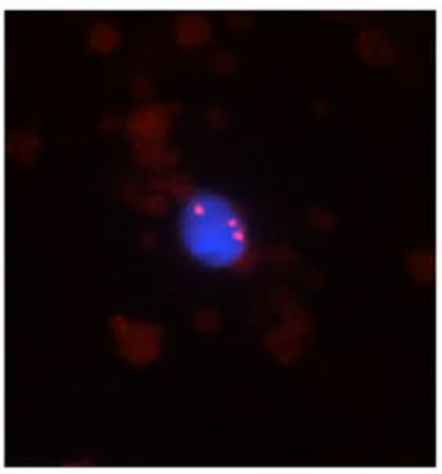

B

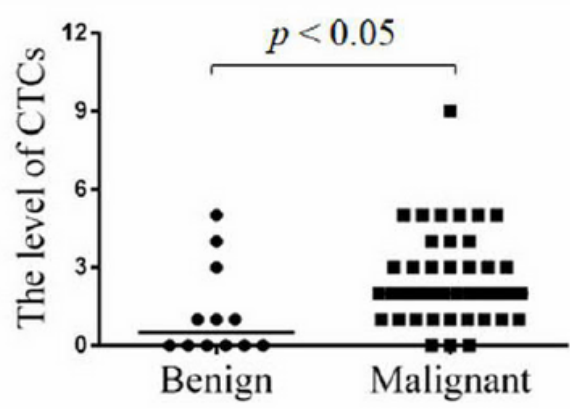

E

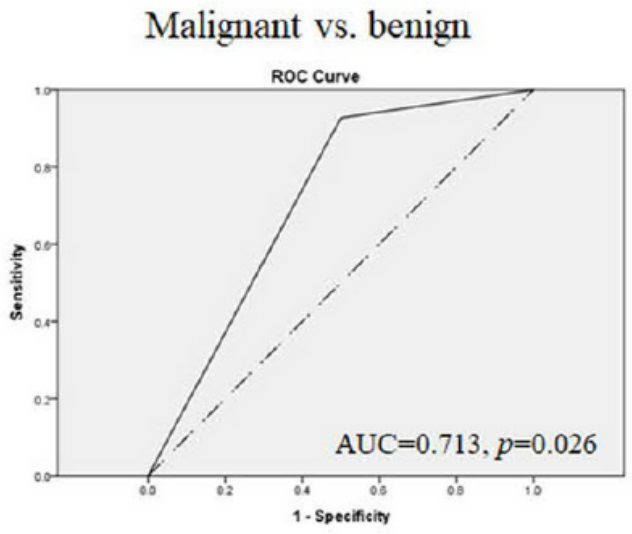

EpCAM

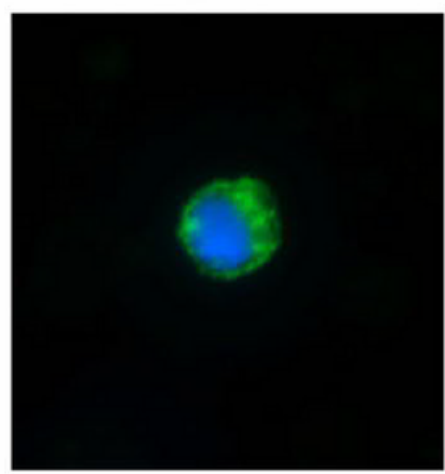

C

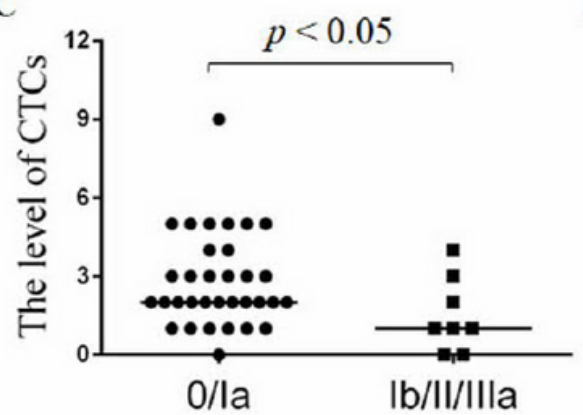

0/la

Pan CK

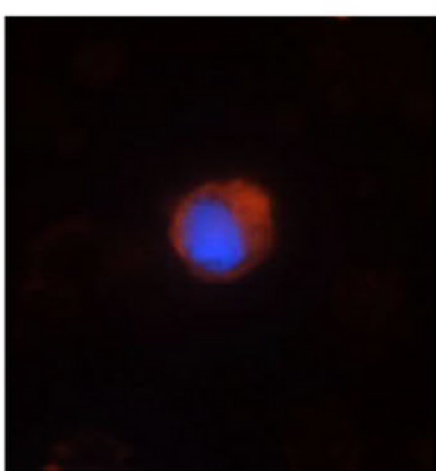

D

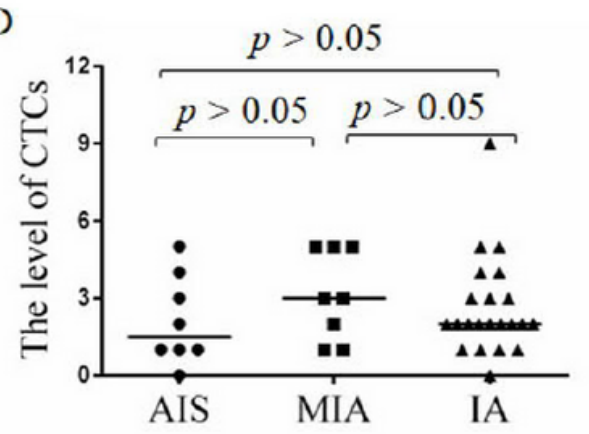

F

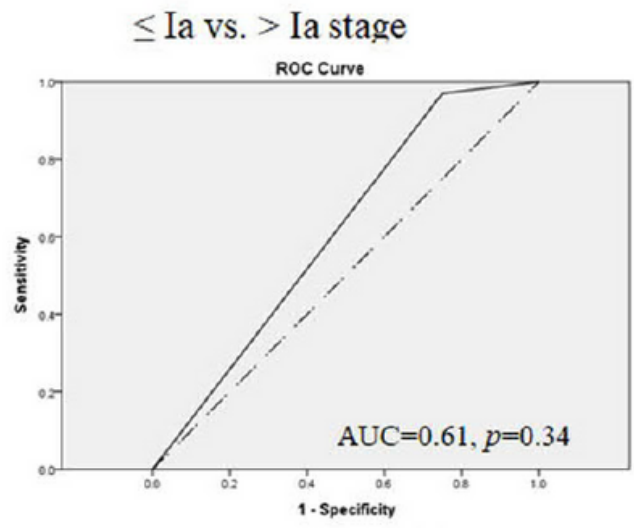

Figure 2

Statistical analysis of the correlation between CTCs and histopathological characteristics of SPNs. A, representative images of circulating tumor cells. CEP8 FISH, EpCAM, pan CKs, DAPI staining of cells enriched from $3.2 \mathrm{~mL}$ peripheral venous blood of patients with SPNs. Magnitude, 200x. B-D, The MannWhitney $U$ test of the CTCs level between different groups as indicated. E and F, The ROC curve of the CTCs level to distingish malignancy from benign $(E)$, or $\leq$ la from $>$ la stage $(F)$. 
A

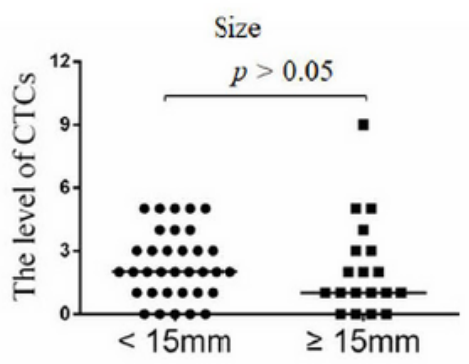

$\mathrm{C}$

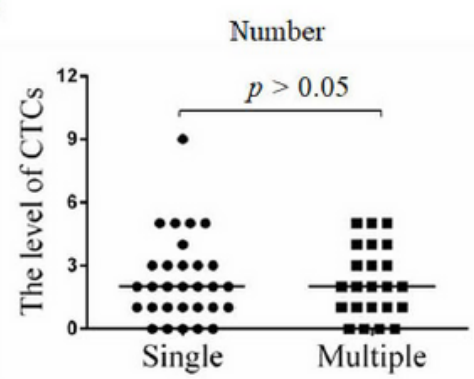

E

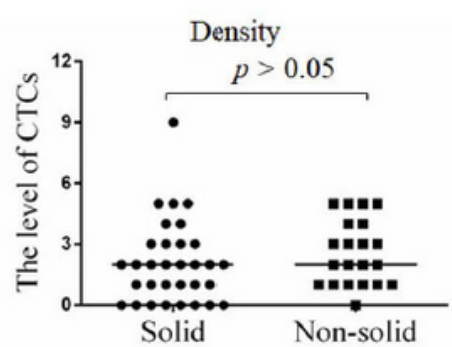

B
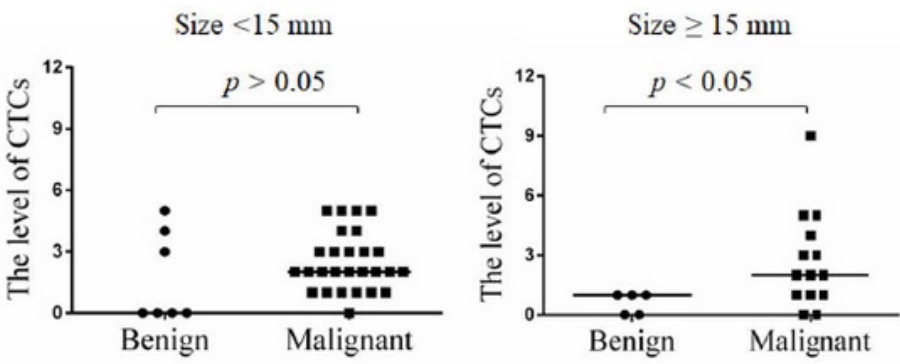

D
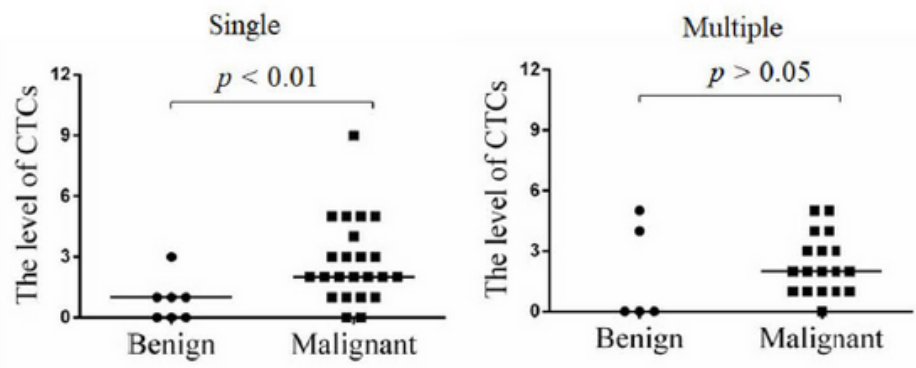

$\mathrm{F}$

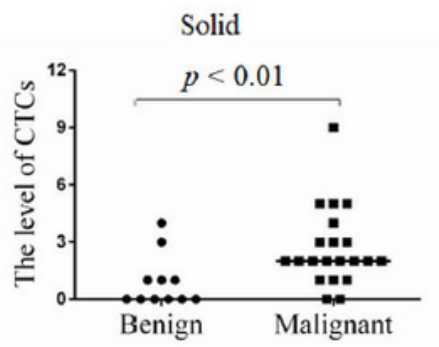

G

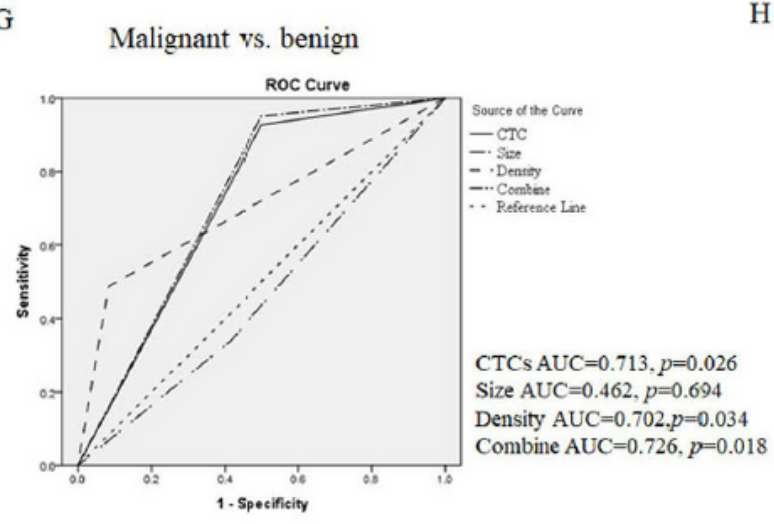

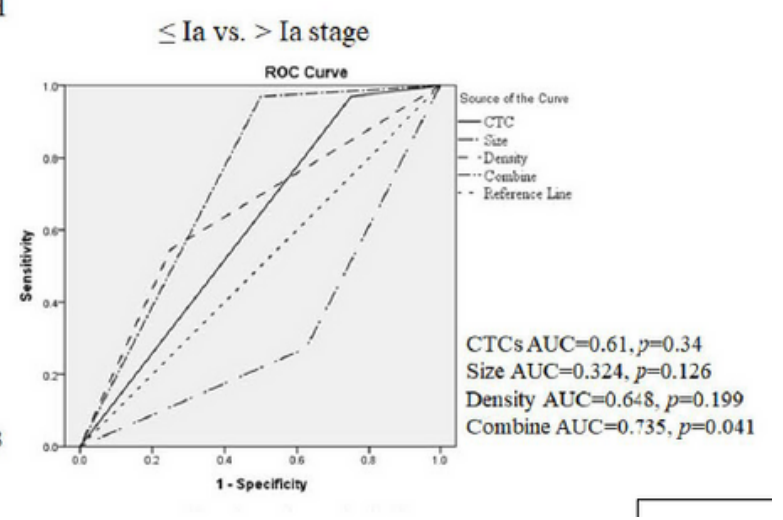

\section{Figure 3}

Statistical analysis of the correlation among CTCs, CT morphological and histopathological characteristics of SPNs. A-F, The Mann-Whitney U test of the CTCs level between different groups as indicated. $\mathrm{G}$ and $\mathrm{H}$, The ROC curve of the CTCs level combined with density to distingish malignancy from benign $(G)$, or $\leq$ la from $>$ la stage $(H)$. 
A

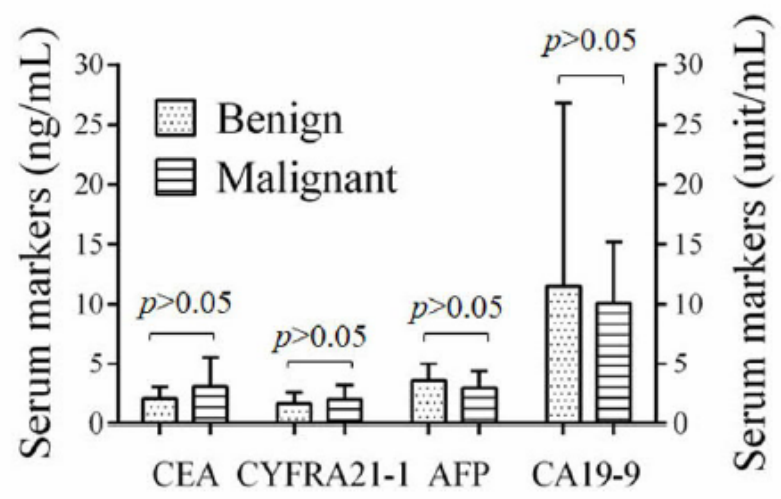

B
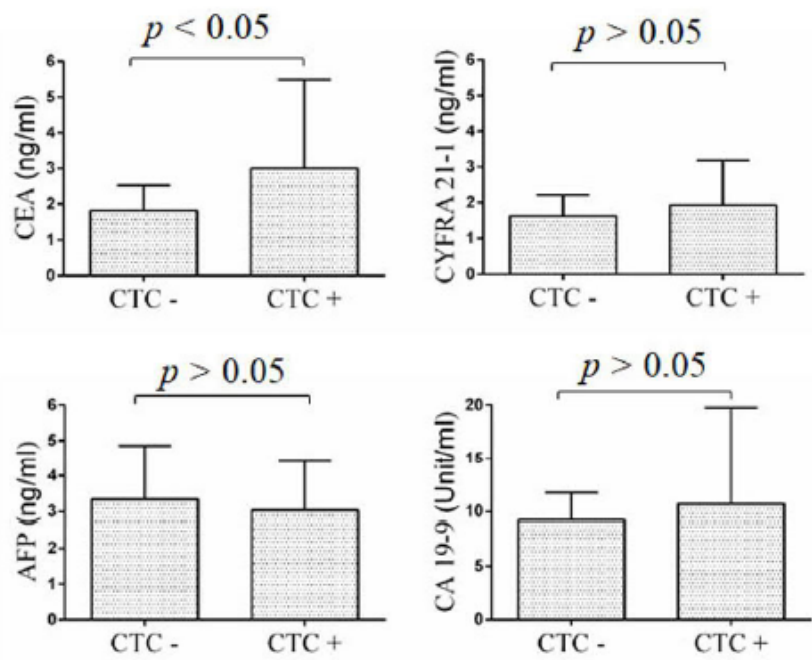

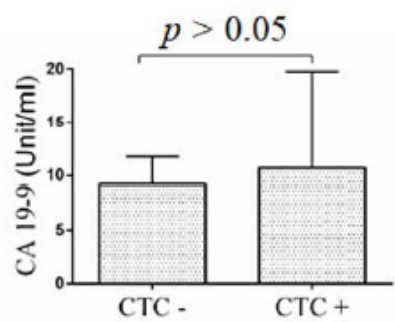

Figure 4

Statistical analysis of the correlation among CTCs, serum markers and histopathological characteristics of SPNs. A, The Student $t$ test of the serum markers between malignant and benign SPNSs. $B$, The student $t$ test of the serum markers between positive and negative CTCs.

\section{Supplementary Files}

This is a list of supplementary files associated with this preprint. Click to download.

- 20200304CTCdataSupplementaryfigures.pdf

- 20200304CTCdataSupplementarytables.pdf 